\title{
Predicting Cancer Risk: Practical Considerations in Developing and Validating a Cancer Risk Prediction Model
}

\author{
Yikyung Park ${ }^{1}$
}

Published online: 14 July 2015

(C) Springer International Publishing AG 2015

\begin{abstract}
As the interest in absolute cancer risk prediction models that estimate the probability of developing cancer has grown steadily, so does the debate on the usefulness of cancer risk prediction models. A newly developed risk prediction model should demonstrate its accuracy, reliability, and generalizability through a validation, preferably using external data. However, a limited number of cancer risk prediction models were independently validated and showed good calibration but weak discriminatory accuracy. Several methods and practical considerations in evaluating and improving cancer risk prediction models' performance are discussed. To increase the utility of cancer risk prediction models in cancer prevention at the population as well as individual level, more powerful and efficient approaches for model development and validation are needed. A collective effort to pool data from cohort studies may provide an excellent opportunity to improve existing models and develop new models, especially for rare cancers and minority populations.
\end{abstract}

Keywords Cancer $\cdot$ Risk prediction model $\cdot$ Absolute risk · Validation $\cdot$ Model performance $\cdot$ Cancer prevention

This article is part of the Topical Collection on Cancer Epidemiology

Yikyung Park

parky@wudosis.wustl.edu

1 Division of Public Health Sciences, Department of Surgery, Washington University School of Medicine, 660 S. Euclid Ave., Campus Box 8100, St. Louis, MO 63110, USA

\section{Introduction}

In 2012, there were an estimated 14.1 million new cancer cases, 8.2 million cancer deaths, and 32.6 million people with cancer within 5 years of diagnosis in the world [1]. The number of new cancer cases is projected to increase to 22.2 million by 2030 [2]. In the United States, cancer overtook heart disease as the leading cause of death in adults aged 40 to 79 years in 2011 [3]. In recent decades, numerous epidemiological studies have confirmed the contribution of environmental and lifestyle factors, such as smoking, diet, alcohol, obesity, and occupational and infectious agents, to the etiology of cancers [4]. Studies have shown that almost $50 \%$ of cancers could be preventable by changing these modifiable risk factors [5-8]. This knowledge on cancer risk factors has been translated into cancer prevention efforts such as smoking cessation to reduce lung cancer and vaccination against hepatitis $B$ virus and human papillomavirus to prevent liver and cervical cancer, respectively. Also, cancer screening that allows early detection of cancer and removal of precancerous lesions has been an effective cancer prevention strategy.

Recently, the National Institutes of Health launched the Precision Medicine Initiative to accelerate the progress of precision medicine. Precision medicine, similar to the terms personalized medicine or stratified medicine, takes individual characteristics in the environment, lifestyle, and genes into account for disease prevention and treatment. One approach to the precision medicine for cancer prevention is to consider an individual's absolute risk of developing a specific type of cancer. An objectively measured absolute risk for cancer can help clinicians and individuals to make an informed decision on cancer prevention strategies, including cancer screening, use of chemopreventive agents, and modification of lifestyle.

A need for cancer risk prediction was recognized in the 1980s, and the first cancer risk prediction model, which was 
for breast cancer, was developed by Gail et al. in 1989 [9, $10 \bullet \cdot$. Since then, a number of risk prediction models for several cancers, including breast, lung, skin, colorectal, ovarian, and other cancers, were developed using various statistical methods [11-24, 25•, 26-28]. Among cancer risk prediction models developed using statistical approaches, some estimate the probability of developing cancer ("absolute risk prediction model"), while others estimate the probability of having a mutation in a cancer susceptibility gene among people with family history of cancer ("genetic susceptibility model") [29-31]. An example of the genetic susceptibility model is the Breast and Ovarian Analysis of Disease Incidence and Carrier Estimation Algorithm (BOADICEA) which estimates $B R C A 1$ and BRCA2 mutation carrier probabilities and agespecific risks of breast and ovarian cancer [32].

Given the steady increase in the interest in cancer risk prediction models, especially for absolute risk prediction, this paper reviews the current status of absolute risk prediction models for cancer and discusses how to improve the models and future opportunities. Most examples used in this paper are breast cancer risk prediction models as they have been extensively studied.

\section{Cancer Risk Prediction: Absolute Risk}

An absolute cancer risk prediction model refers to a statistical tool to estimate an individual's probability of developing cancer over a defined period using the information on multiple risk factors (e.g., behavioral, clinical, genetic factors). To estimate absolute risk, a risk prediction model combines an individual's relative risks for cancer with attributable risks and age-specific cancer incidence rates while accounting for competing risk of death due to other causes [9]. The models are developed using data from cohort, case-control, or clinical studies. A well-known example of an absolute risk prediction model is the Gail breast cancer prediction model [9,33], also known as the Breast Cancer Risk Assessment Tool (BCRAT). The original Gail model was developed using data from white women in the U.S., and risk factors included in the model were age at menarche, age at first live birth, number of breast biopsies, and number of first-degree relatives with breast cancer. According to the BCRAT, a 55-year-old white woman who began menstruating at age 13 years, had first live birth at age 26 years, had no breast biopsies, and whose mother had breast cancer, has a $2.3 \%$ absolute risk of developing breast cancer within the next 5 years and her lifetime risk (until age 90 years) is $15.5 \%$.

An absolute cancer risk prediction model has several applications to cancer prevention. This objective estimate of cancer risk can help individuals to understand his/her risk and may encourage people at high risk to take preventive actions, such as seeking for screening and behavioral changes for modifiable risk factors. An absolute risk is also useful in risk-benefit analyses that can guide people to make a decision whether or not to take more aggressive interventions such as taking tamoxifen to prevent breast cancer [34, 35]. Moreover, risk prediction models can contribute to designing cancer prevention trials by providing eligibility criteria (e.g., eligible for tamoxifen trials if 5 -year breast cancer risk $>1.67 \%$ in the Gail model) and helping statistical power calculations [36, 37], thus improving the efficacy of trials. An absolute risk model has also been used to estimate the population burden of cancer [38] and evaluate cancer prevention strategies (e.g., estimating potential absolute risk reduction by changes in modifiable risk factors) [39]. The usefulness of cancer risk prediction models for cancer prevention at a population level has been well accepted, but its clinical utility in decisionmaking at the individual level has been debated [40].

\section{Statistical Methods of Evaluating the Absolute Risk Prediction Model's Performance}

Before a newly developed risk prediction model becomes widely used, it should demonstrate that the risk prediction model is accurate, reliable, and generalizable. In other words, a model has to be validated. The most commonly used statistical measurements for a model validation are calibration and discrimination. Calibration (i.e., reliability) refers to the agreement between the predicted (i.e., expected $[E]$ number of event) and observed outcomes (i.e., observed $[O]$ number of event) in overall and subgroups of the population. Thus, calibration measures how well the model performs at the population level. A well-calibrated model shows a good agreement, $E / O$ ratio close to 1 . An $E / O$ ratio less than 1 indicates that a model underestimates cancer risk whereas $E / O$ greater than 1 indicates an overestimation of cancer risk. Accurate prediction models also discriminate between an individual who will develop cancer and those who will not develop cancer. Discriminatory accuracy, which shows how well a model performs at the individual level, is commonly assessed by the concordance $(C)$ statistic, also known as the area under the receiver operating characteristic curve (AUC). The value of the AUC corresponds to the probability that a randomly selected individual with cancer has a higher predicted risk than a randomly selected individual without cancer. The AUC value ranges from 0.5 , which indicates no discriminatory power, to 1.0 , perfect discrimination. For example, the AUC of 0.6 means that an individual with cancer had a higher predicted risk than an individual without cancer $60 \%$ of the time overall. The AUC is not the probability that individuals are classified correctly.

When a risk prediction model is evaluated in the same dataset that was used to develop the model, a model is at risk for over-fitting, resulting in an optimistic evaluation of the 
model $[41,42]$. Thus, a model validation is needed to be done in an independent data (i.e., external data) but collected from a population similar to that which the model was derived. However, only a handful of cancer prediction models has been validated in external independent data $[43 \bullet \bullet, 44 \bullet \bullet, 45 \bullet \bullet, 46]$ in part due to limited data availability. A recent systematic review of breast cancer risk prediction models identified 17 breast cancer models but found that only 4 models were validated in independent datasets [43••]. In this study, the Gail breast cancer risk prediction model was the most extensively validated model with 12 validation studies in various populations, including white and black women in the U.S. and women in the UK and Italy as well. The average $E / O$ ratio of the Gail model was 0.95 (95\% confidence interval, CI: $0.88-$ 1.01 ), and the average AUC was 0.63 (95\% CI: 0.59-0.67). Another example, a colorectal cancer risk prediction model developed by Freedman et al.[22] was validated in an external data. The $E / O$ ratio of this colorectal cancer risk prediction model was 0.99 (95\% CI: 0.95-1.04) in men and 1.05 (95 \% CI: 0.98-1.11) in women, and the AUC value was 0.61 in both men and women [47]. As seen in these examples

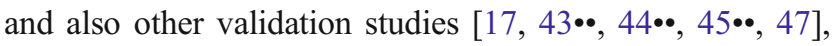
cancer risk prediction models validated in external data were generally well calibrated, but they had a weak discriminatory accuracy. In other words, cancer risk prediction models worked reasonably well at predicting at the population level but not at the individual level. Due to low discriminatory accuracy, the usefulness of a cancer risk prediction model as a clinical decision-making tool for screening has been debated.

However, the evaluation of risk prediction models' performance should not be solely based on the assessment of calibration and discriminatory accuracy [48•]. The AUC, commonly used to assess discriminatory accuracy, does not indicate how well a risk prediction model can separate individuals with low risk from those with high risk, which may be more meaningful in clinical decision-making. To assess how well a risk prediction model classifies individuals into appropriate risk groups, a reclassification table that cross-tabulates absolute risks estimated from models with and without the new risk factors was proposed [49]. In addition, a net reclassification index (NRI) and an integrated discrimination index were introduced to estimate the differences in classification of the population into high- and low-risk categories [48•, 50, 51]. The NRI is the sum of differences in proportions of individuals moving to a higher risk category minus the proportion of individuals moving to a lower risk category for those with the outcome and the proportion of individuals moving to a lower risk category minus the proportion of individuals moving to a higher risk category for those without the outcome [50]. A study that evaluated the Gail breast cancer risk prediction model with single nucleotide polymorphisms (SNPs) and without SNPs showed how a reclassification table and the
NRI can be used [52•]. This study found that when women were categorized into low- $(<1.5 \%)$, intermediate- $(1.5-$ $2.0 \%)$, and high-risk groups $(>2.0 \%)$ based on a 5 -year absolute risk, the model with SNPs classified $22 \%$ fewer women with breast cancer and $29 \%$ fewer women without breast cancer into the intermediate group compared with the model without SNPs. The classification also improved for $5.6 \%$ of women with breast cancer and $2.9 \%$ of women without breast cancer, showing an NRI value of 0.085 ( $p$ value $=1.0 \times 10^{-5}$ ).

\section{Comparing Multiple Cancer Risk Prediction Models' Performance}

As several risk prediction models exist for one cancer site, a systematic review of existing models would be a good opportunity to compare models' performance. Few systematic reviews and meta-analyses were conducted to summarize and compare cancer risk prediction models for breast and colorectal cancer $[43 \bullet \bullet, 44 \bullet \cdot, 46]$. The authors of these reviews reported that one of the challenges of conducting a systematic review was the difficulties in extracting the necessary information for review due to a lack of consistency in reporting across studies. For example, some studies did not report basic study information, such as a number of cases and the amount of missing data, while others did not provide enough details on statistical methods. Set aside a limitation in quantitative comparisons using measures of calibration and discriminatory accuracy across studies, it is not possible to conduct a qualitative review of risk prediction models without some consistently reported information. These review papers strongly supported a need for standards for reporting risk prediction models. As more studies are expected to publish cancer risk prediction models, a guideline for reporting a cancer risk prediction model is urgently needed.

Although systematic reviews and meta-analyses are useful in comparing and summarizing multiple risk prediction models for one cancer, a head-to-head comparison that evaluates all models in one external dataset would be of great interest, but it is very rare. There is one study that compared the performance of three lung cancer risk prediction models in the same population [45••]. These lung cancer risk prediction models used different sets of risk factors. There were some common risk factors (e.g., smoking, asbestos) but many other different risk factors (e.g., family history, respiratory diseases) were used in each model. When the three models were compared for their discriminatory accuracy and positive and negative predictive values, these models showed similar discriminatory accuracy (AUC ranged from 0.66 to 0.69 ), but depending on the evaluation criteria, one model performed better than others did. This study demonstrates that no one model outperforms the others and each model still needs to 
be improved. More studies evaluating several risk prediction models simultaneously in one dataset are needed.

\section{Improving Absolute Risk Prediction Model: Validating a Model Over Time and in Various Populations}

The performance of an absolute risk prediction model changes over time because of changes in underlying cancer incidence in a population. The Gail breast cancer model was first developed in 1989 using the breast cancer incidence rates and competing risks from 1983 to 1987 in the U.S. [9]. Since then, the breast cancer incidence rates in the U.S. had increased during the 1990s, declined moderately starting in 2000, and substantially dropped in 2003 [53]. When the original Gail model was used to estimate women's risk in studies conducted between mid-1990s and mid-2000s, the model underpredicted the number of expected breast cancer cases by $13-14 \%$ in white women [54-] and $18 \%$ in Hispanic women [55]. However, after the Gail model was updated with breast cancer incidence rates and competing mortality rates from 1995 to 2003, reflecting contemporary studies' timeline, the model's performance, in terms of calibration, improved [54•, 55].

A performance of a risk prediction model also varies by the population in which the model was evaluated in. Differences in demographic characteristics (e.g., race/ethnicity, age distribution), the prevalence of risk factors, and the strengths of associations (i.e., relative risks) between risk factors and cancer may affect a model's performance across populations. Race/ethnic differences should be carefully examined because cancer incidence rates, prevalence of risk factors, and the strength of relative risks may differ by race/ethnic groups. The Gail model that was developed using data from white women in 1989 was evaluated in Hispanic women in the Women's Health Initiative study conducted in 1993-2007 [55]. Updating the Gail model with contemporary breast cancer incidence rate (1993-2007) improved the calibration of the Gail model overall in Hispanic women. However, breast cancer risk was significantly underestimated in Hispanic women with breast biopsies. It may be due to a significant difference in the relative risk for number of breast biopsies in Hispanic women $(\mathrm{RR}=1.71)$ and in white women $(\mathrm{RR}=1.27, p$ for difference between Hispanic women and white women= $0.03)$. The Gail model also did not perform well when it was evaluated in African American women [56]. Again, the relative risks for number of biopsies in African American women (e.g., $R R$ for $\geq 2$ biopsies in women $<50$ years old $=1.44$ ) were lower than those from the Gail model $(\mathrm{RR}=2.88)$, and this may contribute to a significant underestimation of breast cancer risk in African American women who had breast biopsies. Therefore, the CARE model, a modified Gail model using African American women data from a breast cancer case- control study, was developed [56]. Recently, another breast cancer risk prediction model for African Americans was developed using data from a large prospective cohort of African Americans [25•]. This new breast cancer risk prediction model for African Americans included several new risk factors, such as body mass index at age 18, height, oral contraceptive use, and bilateral oophorectomy, which were found to be risk factors for breast cancer in this cohort of African American women. These examples demonstrate that a risk prediction model developed in a particular race/ethnic group may not be directly applied to other race/ethnic groups. An inappropriate model will provide an inaccurate probability of developing cancer to an individual, which may result in misrepresentation of a specific race/ethnic group in cancer screening or prevention trials.

\section{Improving the Absolute Risk Prediction Model: Risk Factors and Outcome}

The most common approach to improve a risk prediction model's performance is updating the model by adding new risk factors. Several attempts were made to incorporate newly identified cancer risk factors, mainly clinical and genetic factors, into an existing risk prediction model. For example, a mammographic density of the breast was not included in the original Gail breast cancer prediction model developed in 1989 (AUC=0.60) [18]. When the mammographic density was added to the Gail model, the risk prediction model showed a slight improvement in discriminatory accuracy (AUC $=0.64-0.66)[18,57]$. A lung cancer risk prediction model developed by Spitz et al. was also expanded by adding two new biomarkers for lung cancer risk, DNA repair capacity and bleomycin sensitivity [58]. The updated model with these two new biomarkers slightly increased the AUC from 0.68 to 0.70 in current smokers. Although these studies $[18,57,58]$ showed that the use of clinical or biomarkers could improve a risk prediction model's performance, it may not feasible to use biomarkers in large-scale population studies unless those biomarkers can be measured cost-effectively by standardized methods and routinely used in a clinical setting.

The utility of genetic information in a cancer prediction model has also been debated. Studies found that an inclusion of SNPs in a cancer risk prediction model did not significantly improve a model's discriminatory ability [16, 59-61]. The AUC increased modestly from 0.58 to 0.62 when ten SNPs were added to a breast cancer risk prediction model [59]. In a pancreatic cancer risk prediction model, the NRI comparing the model with three SNPs to the model without SNPs was $-0.01(p=0.89)$ in men and $-0.02(p=0.97)$ in women [16]. Furthermore, a study that investigated the usefulness of foreseeable (to be discovered) SNPs in a cancer risk prediction model found that even the most optimistic foreseeable models 
may not improve risk prediction models' discriminatory accuracy [61]. Moreover, no studies were able to consider potential gene-gene or gen-environmental interactions in a cancer risk prediction model. Even though genetic testing becomes more acceptable, if genetic testing is required to estimate absolute risk, it may not be practical for the risk prediction model to be used in a large population-based screening or preventive interventions. Furthermore, if an absolute cancer risk prediction model (not a genetic susceptibility model) uses mainly genetic information and few lifestyle factors, it may also have unintended consequences due to people's misperception. Some people may interpret that their cancer risk is largely driven by genes that cannot be changed, and efforts to change their unhealthy behavior would not help them to lower their cancer risk.

When potential risk factors, often clinical or biomarkers, are measured in a subset of the study population only, the multiple imputation method [62] may be useful. To evaluate if an inclusion of estradiol levels improves the Rosner-Colditz breast cancer risk prediction model, Rosner used the estradiol data collected in a subset of women in the prospective cohort study that the model was originally developed with [63]. Estradiol concentration was imputed for all women using the available data from the subset. When the imputed estradiol was added to the Rosner-Colditz breast cancer risk prediction model using the multiple imputation method, the AUC slightly increased from 0.635 to $0.645(p<0.001)$. Rosner also evaluated the utility of imputed future use of menopausal hormone therapy (MHT) in the Rosner-Colditz breast cancer risk prediction model [64•]. Using the prospective cohort study which collected MHT use every 2 years during follow-up, the performance of the model with imputed future use of MHT (AUC $=0.600)$ was similar to that of the model with observed MHT use data every 2 years (overall AUC $=0.616$ ). The imputed MHT use was also used in the validation of the Rosner-Colditz model in an independent dataset which did not have the updated MHT every 2 years. These studies showed that the imputation method may be useful in model development and validation when some risk factors are not available in the whole study population or in a validation dataset.

A risk prediction model's performance may be improved by accounting for differences in cancer subtypes. Accumulating evidence suggests that risk factors and incidence rates differed by their subtype (e.g., estrogen receptor positive breast cancer vs. estrogen receptor negative breast cancer) [65-67]. Therefore, modeling each cancer subtype separately may improve a model's discriminatory accuracy. A colorectal cancer risk prediction model developed by Freedman et al. applied this approach and estimated relative risks for each subtype (proximal colon, distal colon, and rectum) separately and also used subtype-specific incidence rates [22].

\section{Opportunities in Cancer Risk Prediction Model}

One of the significant barriers in developing and validating a cancer risk prediction model is a lack of data that has a large number of cancer cases and contains all potential risk factors. Although cancer is the leading cause of deaths, cancer is still a rare disease. Even a large population-based cancer study with more than 50,000 participants and several decades of followup does not have enough number of cancer cases, especially for less frequent cancers such as ovarian, pancreatic, and kidney cancer. A recent trend in collaboration through consortia, especially for prospective cohort studies, provides an excellent opportunity for a development and a validation of cancer risk prediction models. Pooled data from several large prospective cohorts will provide data with various population mixes, which are needed to develop and validate a cancer risk model that is generalizable to most race/ethnic groups. Pooling data from several studies is critical to develop a risk prediction model for less frequent cancers and minority populations. Also, the pooled data may allow the incorporation of interactions between risk factors (e.g., interaction between obesity and hormone replacement therapy) which have not been well examined in previous models. Furthermore, a linkage of cohort members to electronic health records or administrative health claims data (e.g., Medicare) may provide a robust dataset supplemented with various clinical information.

Another challenge in developing a cancer risk prediction model is that our knowledge on cancer risk factors and disease pathways is limited. To improve a cancer risk prediction model's performance, new cancer risk factors, including environmental, behavioral, and clinical information, are needed. Risk factors used in currently available cancer risk prediction models are mostly mid- to late-adulthood risk factors. Accumulating evidence suggests that earlier in the life course, such as childhood and adolescence, is a critical period [68], but little is known. More efforts to identify cancer risk factors across the entire spectrum of the life course are needed. Also, the efforts to find new molecular and clinical biomarkers should be continued. Behavioral risk factors that can be easily and also reliably obtained from individuals and biomarkers that can be measured cost-effectively in routine practice are likely to be good candidate risk factors for cancer risk prediction models.

\section{Conclusions}

As the number of cancer risk prediction models has increased steadily, so has the interest in using a risk prediction model for cancer prevention practice at the population as well as the individual level. However, a lack of independent validations of cancer risk prediction models significantly restricts the 
utility of a risk prediction model. More vigorous validations of each risk prediction model in independent data and head-tohead comparisons of multiple risk prediction models in the same population are urgently needed. To improve the performance of a cancer risk prediction model, each step of the model development, from the selection of risk factors to heterogeneities in cancer subtypes, and study populations, should be carefully examined. Also, efforts to incorporate newly identified cancer risk factors into the model should be continued. However, a risk prediction model intended for common use by individuals and clinicians would use risk factors that can be easily obtained from individuals and measured cost-effectively in routine practice. A lack of consistency in reporting cancer risk prediction models in published studies significantly hinders efforts to understand each model and compare existing multiple risk prediction models for one cancer site. A guideline for reporting the results of risk prediction model development and validation studies is warranted to improve the quality of reporting. Finally, a collective effort to pool data from cohort studies for the development and validation of cancer risk prediction models, especially for rare cancers and minority populations, is strongly encouraged.

\section{Compliance with Ethics Guidelines}

\section{Conflict of Interest Y Park declares no conflicts of interest.}

Human and Animal Rights and Informed Consent All studies by $Y$ Park involving animal and/or human subjects were performed after approval by the appropriate institutional review boards. When required, written informed consent was obtained from all participants.

\section{References}

1. Ferlay J, Soerjomataram I, Dikshit R, Eser S, Mathers C, Rebelo M, et al. Cancer incidence and mortality worldwide: sources, methods and major patterns in GLOBOCAN 2012. Int J Cancer J Int Cancer. 2015;136(5):E359-86.

2. Bray F, Jemal A, Grey N, Ferlay J, Forman D. Global cancer transitions according to the human development index (2008-2030): a population-based study. Lancet Oncol. 2012;13(8):790-801.

3. Siegel RL, Miller KD, Jemal A. Cancer statistics, 2015. CA Cancer J Clin. 2015;65(1):5-29.

4. Schottenfeld D, Fraumeni JF. Cancer epidemiology and prevention. 3rd ed. New York: Oxford University Press; 2006.

5. Colditz GA, Sellers TA, Trapido E. Epidemiology - identifying the causes and preventability of cancer? Nat Rev Cancer. 2006;6(1): 75-83.

6. Doll R, Peto R. The causes of cancer: quantitative estimates of avoidable risks of cancer in the United States today. J Natl Cancer Inst. 1981;66(6):1191-308.

7. Parkin DM, Boyd L, Walker LC. 16. The fraction of cancer attributable to lifestyle and environmental factors in the UK in 2010. Br J Cancer. 2011;105 Suppl 2:S77-81.
8. Colditz GA, Wei EK. Preventability of cancer: the relative contributions of biologic and social and physical environmental determinants of cancer mortality. Annu Rev Public Health. 2012;33:137-56

9. Gail MH, Brinton LA, Byar DP, Corle DK, Green SB, Schairer C, et al. Projecting individualized probabilities of developing breast cancer for white females who are being examined annually. J Natl Cancer Inst. 1989;81(24):1879-86.

$10 . \bullet$ Gail MH. Twenty-five years of breast cancer risk models and their applications. J Natl Cancer Inst. 2015;107(5). Reviewed the Gail breast cancer risk prediction model and its applications and discussed the prospects for a model improvement

11. Fears TR, Guerry D, Pfeiffer RM, Sagebiel RW, Elder DE, Halpern A, et al. Identifying individuals at high risk of melanoma: a practical predictor of absolute risk. J Clin Oncol. 2006;24(22):3590-6.

12. Cho E, Rosner BA, Feskanich D, Colditz GA. Risk factors and individual probabilities of melanoma for whites. J Clin Oncol. 2005;23(12):2669-75.

13. Cassidy A, Myles JP, van Tongeren M, Page RD, Liloglou T, Duffy $\mathrm{SW}$, et al. The LLP risk model: an individual risk prediction model for lung cancer. Br J Cancer. 2008;98(2):270-6.

14. Bach PB, Kattan MW, Thornquist MD, Kris MG, Tate RC, Barnett MJ, et al. Variations in lung cancer risk among smokers. J Natl Cancer Inst. 2003;95(6):470-8.

15. Tammemagi CM, Pinsky PF, Caporaso NE, Kvale PA, Hocking WG, Church TR, et al. Lung cancer risk prediction: prostate, lung, colorectal and ovarian cancer screening trial models and validation. J Natl Cancer Inst. 2011;103(13):1058-68.

16. Klein AP, Lindstrom S, Mendelsohn JB, Steplowski E, Arslan AA, Bueno-de-Mesquita HB, et al. An absolute risk model to identify individuals at elevated risk for pancreatic cancer in the general population. PLoS One. 2013;8(9), e72311.

17. Pfeiffer RM, Park Y, Kreimer AR, Lacey Jr JV, Pee D, Greenlee RT, et al. Risk prediction for breast, endometrial, and ovarian cancer in white women aged 50 y or older: derivation and validation from population-based cohort studies. PLoS Med. 2013;10(7), e1001492.

18. Chen J, Pee D, Ayyagari R, Graubard B, Schairer C, Byrne C, et al. Projecting absolute invasive breast cancer risk in white women with a model that includes mammographic density. J Natl Cancer Inst. 2006;98(17):1215-26.

19. Rosner B, Colditz GA. Nurses' health study: log-incidence mathematical model of breast cancer incidence. J Natl Cancer Inst. 1996;88(6):359-64.

20. Colditz GA, Rosner B. Cumulative risk of breast cancer to age 70 years according to risk factor status: data from the Nurses' Health Study. Am J Epidemiol. 2000;152(10):950-64.

21. Barlow WE, White E, Ballard-Barbash R, Vacek PM, TitusErnstoff L, Carney PA, et al. Prospective breast cancer risk prediction model for women undergoing screening mammography. J Natl Cancer Inst. 2006;98(17):1204-14.

22. Freedman AN, Slattery ML, Ballard-Barbash R, Willis G, Cann BJ, Pee D, et al. Colorectal cancer risk prediction tool for white men and women without known susceptibility. J Clin Oncol. 2009;27(5):686-93.

23. Wei EK, Colditz GA, Giovannucci EL, Fuchs CS, Rosner BA. Cumulative risk of colon cancer up to age 70 years by risk factor status using data from the Nurses' Health Study. Am J Epidemiol. 2009;170(7):863-72.

24. Spitz MR, Hong WK, Amos CI, Wu X, Schabath MB, Dong Q, et al. A risk model for prediction of lung cancer. J Natl Cancer Inst. 2007;99(9):715-26. 
25. Boggs DA, Rosenberg L, Adams-Campbell LL, Palmer JR. Prospective approach to breast cancer risk prediction in African american women: the black women's health study model. J Clin Oncol. 2015;33(9):1038-44. Majority of cancer risk prediction model developed in the U.S. are based on white population. But this study developed a breast cancer risk prediction model for African Americans using data from African Americans and suggested a need for a population specific cancer model.

26. Husing A, Dossus L, Ferrari P, Tjonneland A, Hansen L, Fagherazzi $\mathrm{G}$, et al. An epidemiological model for prediction of endometrial cancer risk in Europe. Eur J Epidemiol. 2015.

27. Li K, Husing A, Fortner RT, Tjonneland A, Hansen L, Dossus L, et al. An epidemiologic risk prediction model for ovarian cancer in Europe: the EPIC study. Br J Cancer. 2015;112(7):1257-65.

28. Rosner BA, Colditz GA, Webb PM, Hankinson SE. Mathematical models of ovarian cancer incidence. Epidemiology (Cambridge, Mass). 2005;16(4):508-15.

29. Balmana J, Stockwell DH, Steyerberg EW, Stoffel EM, Deffenbaugh AM, Reid JE, et al. Prediction of MLH1 and MSH2 mutations in Lynch syndrome. JAMA. 2006;296(12):1469-78.

30. Niendorf KB, Goggins W, Yang G, Tsai KY, Shennan M, Bell DW, et al. MELPREDICT: a logistic regression model to estimate CDKN2A carrier probability. J Med Genet. 2006;43(6):501-6.

31. Wang W, Niendorf KB, Patel D, Blackford A, Marroni F, Sober AJ, et al. Estimating CDKN2A carrier probability and personalizing cancer risk assessments in hereditary melanoma using MelaPRO. Cancer Res. 2010;70(2):552-9.

32. Lee AJ, Cunningham AP, Kuchenbaecker KB, Mavaddat N, Easton DF, Antoniou AC. BOADICEA breast cancer risk prediction model: updates to cancer incidences, tumour pathology and web interface. Br J Cancer. 2014;110(2):535-45.

33. Costantino JP, Gail MH, Pee D, Anderson S, Redmond CK, Benichou J, et al. Validation studies for models projecting the risk of invasive and total breast cancer incidence. J Natl Cancer Inst. 1999;91(18):1541-8.

34. Gail MH, Costantino JP, Bryant J, Croyle R, Freedman L, Helzlsouer $\mathrm{K}$, et al. Weighing the risks and benefits of tamoxifen treatment for preventing breast cancer. J Natl Cancer Inst. 1999;91(21):1829-46.

35. Freedman AN, Yu B, Gail MH, Costantino JP, Graubard BI, Vogel $\mathrm{VG}$, et al. Benefit/risk assessment for breast cancer chemoprevention with raloxifene or tamoxifen for women age 50 years or older. J Clin Oncol. 2011;29(17):2327-33.

36. Fisher B, Costantino JP, Wickerham DL, Redmond CK, Kavanah M, Cronin WM, et al. Tamoxifen for prevention of breast cancer: report of the national surgical adjuvant breast and bowel project P-1 study. J Natl Cancer Inst. 1998;90(18):1371-88.

37. Vogel VG, Costantino JP, Wickerham DL, Cronin WM, Cecchini RS, Atkins JN, et al. Effects of tamoxifen vs raloxifene on the risk of developing invasive breast cancer and other disease outcomes: the NSABP study of tamoxifen and raloxifene (STAR) P-2 trial. Jama. 2006;295(23):2727-41.

38. Graubard BI, Freedman AN, Gail MH. Five-year and lifetime risk of breast cancer among U.S. subpopulations: implications for magnetic resonance imaging screening. Cancer Epidemiol Biomark Prev. 2010;19(10):2430-6.

39. Petracci E, Decarli A, Schairer C, Pfeiffer RM, Pee D, Masala G, et al. Risk factor modification and projections of absolute breast cancer risk. J Natl Cancer Inst. 2011;103(13):1037-48.

40. Vickers AJ. Prediction models in cancer care. CA Cancer J Clin. 2011.

41. Bleeker SE, Moll HA, Steyerberg EW, Donders AR, DerksenLubsen G, Grobbee DE, et al. External validation is necessary in prediction research: a clinical example. J Clin Epidemiol. 2003;56(9):826-32.
42. Steyerberg EW. Clinical prediction models: a practical approach to development, validation, and updating. Springer. 2009.

43.• Meads C, Ahmed I, Riley RD. A systematic review of breast cancer incidence risk prediction models with meta-analysis of their performance. Breast Cancer Res Treat. 2012;132(2):365-77. Systematically evaluated multiple breast cancer risk prediction models and performed meta-analyses of measures of calibration and discriminatory accuracy reported in studies. The study supports a need for a standard format for reporting risk prediction models across studies.

44.• Anothaisintawee T, Teerawattananon Y, Wiratkapun C, Kasamesup V, Thakkinstian A. Risk prediction models of breast cancer: a systematic review of model performances. Breast Cancer Res Treat. 2012;133(1):1-10. Systematically reviewed existing breast cancer risk prediction models and showed a need for a standard format for reporting risk prediction models across studies.

45.• D'Amelio Jr AM, Cassidy A, Asomaning K, Raji OY, Duffy SW, Field JK, et al. Comparison of discriminatory power and accuracy of three lung cancer risk models. Br J Cancer. 2010;103(3):423-9. Compared three different lung cancer risk prediction models by evaluating them in one external data.

46. Win AK, Macinnis RJ, Hopper JL, Jenkins MA. Risk prediction models for colorectal cancer: a review. Cancer Epidemiol Biomark Prev. 2012;21(3):398-410.

47. Park Y, Freedman AN, Gail MH, Pee D, Hollenbeck A, Schatzkin A, et al. Validation of a colorectal cancer risk prediction model among white patients age 50 years and older. J Clin Oncol. 2009;27(5):694-8.

48. Steyerberg EW, Vickers AJ, Cook NR, Gerds T, Gonen M, Obuchowski N, et al. Assessing the performance of prediction models: a framework for traditional and novel measures. Epidemiology (Cambridge, Mass). 2010;21(1):128-38. Reviewed classic and novel methods of evaluating the performance of risk prediction models.

49. Cook NR. Use and misuse of the receiver operating characteristic curve in risk prediction. Circulation. 2007;115(7):928-35.

50. Pencina MJ, D'Agostino Sr RB, D'Agostino Jr RB, Vasan RS. Evaluating the added predictive ability of a new marker: from area under the ROC curve to reclassification and beyond. Stat Med. 2008;27(2):157-72. discussion 207-112.

51. Janes H, Pepe MS, Gu W. Assessing the value of risk predictions by using risk stratification tables. Ann Intern Med. 2008;149(10):751-60.

52. Mealiffe ME, Stokowski RP, Rhees BK, Prentice RL, Pettinger M, Hinds DA. Assessment of clinical validity of a breast cancer risk model combining genetic and clinical information. J Natl Cancer Inst. 2010;102(21):1618-27. Showed a use of new approaches to evaluate the performance of a risk prediction model.

53. Glass AG, Lacey Jr JV, Carreon JD, Hoover RN. Breast cancer incidence, 1980-2006: combined roles of menopausal hormone therapy, screening mammography, and estrogen receptor status. J Natl Cancer Inst. 2007;99(15):1152-61.

54. Schonfeld SJ, Pee D, Greenlee RT, Hartge P, Lacey Jr JV, Park Y, et al. Effect of changing breast cancer incidence rates on the calibration of the gail model. J Clin Oncol. 2010;28(14):2411-7. Showed an importance of updating a cancer risk prediction model over time.

55. Banegas MP, Gail MH, LaCroix A, Thompson B, Martinez ME, Wactawski-Wende J, et al. Evaluating breast cancer risk projections for Hispanic women. Breast Cancer Res Treat. 2012;132(1): $347-53$.

56. Gail MH, Costantino JP, Pee D, Bondy M, Newman L, Selvan M, et al. Projecting individualized absolute invasive breast cancer risk in African American women. J Natl Cancer Inst. 2007;99(23): 1782-92. 
57. Tice JA, Cummings SR, Smith-Bindman R, Ichikawa L, Barlow WE, Kerlikowske K. Using clinical factors and mammographic breast density to estimate breast cancer risk: development and validation of a new predictive model. Ann Intern Med. 2008;148(5): 337-47.

58. Spitz MR, Etzel CJ, Dong Q, Amos CI, Wei Q, Wu X, et al. An expanded risk prediction model for lung cancer. Cancer Prev Res (Phila). 2008;1(4):250-4.

59. Wacholder S, Hartge P, Prentice R, Garcia-Closas M, Feigelson HS, Diver WR, et al. Performance of common genetic variants in breastcancer risk models. N Engl J Med. 2010;362(11):986-93.

60. Aschard H, Chen J, Cornelis MC, Chibnik LB, Karlson EW, Kraft $\mathrm{P}$. Inclusion of gene-gene and gene-environment interactions unlikely to dramatically improve risk prediction for complex diseases. Am J Hum Genet. 2012;90(6):962-72.

61. Park JH, Gail MH, Greene MH, Chatterjee N. Potential usefulness of single nucleotide polymorphisms to identify persons at high cancer risk: an evaluation of seven common cancers. J Clin Oncol. 2012;30(17):2157-62.

62. Rubin D. Multiple imputation for nonresponse in surveys 1987. New York: Wiley; 1987.

63. Rosner B, Colditz GA, Iglehart JD, Hankinson SE. Risk prediction models with incomplete data with application to prediction of estrogen receptor-positive breast cancer: prospective data from the Nurses' Health Study. Breast Cancer Res. 2008;10(4):R55.

64. Rosner BA, Colditz GA, Hankinson SE, Sullivan-Halley J, Lacey Jr JV, Bernstein L. Validation of Rosner-Colditz breast cancer incidence model using an independent data set, the California Teachers Study. Breast Cancer Res Treat. 2013;142(1):187-202. Showed a use of the imputation method in a risk model development and validation.

65. Colditz GA, Rosner BA, Chen WY, Holmes MD, Hankinson SE. Risk factors for breast cancer according to estrogen and progesterone receptor status. J Natl Cancer Inst. 2004;96(3):218-28.

66. Wei EK, Giovannucci E, Wu K, Rosner B, Fuchs CS, Willett WC, et al. Comparison of risk factors for colon and rectal cancer. Int $\mathrm{J}$ Cancer J Int Cancer. 2004;108(3):433-42.

67. Yang HP, Trabert B, Murphy MA, Sherman ME, Sampson JN, Brinton LA, et al. Ovarian cancer risk factors by histologic subtypes in the NIH-AARP diet and health study. Int J Cancer J Int Cancer. 2012;131(4):938-48.

68. Colditz GA, Frazier AL. Models of breast cancer show that risk is set by events of early life: prevention efforts must shift focus. Cancer Epidemiol Biomark Prev. 1995;4(5):567-71. 